\title{
EXTREME POSITIVE LINEAR MAPS \\ BETWEEN JORDAN BANACH ALGEBRAS
}

\author{
Cho-Ho Chu and Nigel P.H. Jefferies
}

Let $A$ and $B$ be unital JB-algebras. We study the extremal structure of the convex set $S(A, B)$ of all identity preserving positive linear maps from $A$ to $B$. We show that every unital Jordan homomorphism from $A$ to $B$ is an extreme point of $S(A, B)$. An extreme point of $S(A, B)$ need not be a homomorphism and we show that, given $A$, every extreme point of $S(A, B)$ is a homomorphism for any $B$ if, and only if, $\operatorname{dim} A \leq 2$. We also determine when $S(A, B)$ is a simplex.

\section{Introduction}

Let $A$ and $B$ be unital JB-algebras. In this paper, we study the extreme points of the convex set $S(A, B)$ of all identity preserving positive linear maps from $A$ to $B$.

Motivated by the results in $C^{\star}$-algebras $[2,4,10]$, we begin by showing that every unital Jordan homomorphism from $A$ to $B$ is an extreme point of $S(A, B)$. We then focus our attention on the natural question of the converse. We study conditions under which the extreme points of $S(A, B)$ are Jordan homomorphisms. If $A$ and $B$ are associative, it is known that the extreme points of $S(A, B)$ are exactly the unital

Received 19 May 1986.

Copyright Clearance Centre, Inc. Serial-fee code: 0004-9727/87 $\$ A 2.00+0.00$. 
homomorphisms from $A$ to $B$. In the nonassociative case, however, our results indicate that only in very special situations can one expect that every extreme point of $S(A, B)$ is a homomorphism. For instance, given $A$, every extreme point of $S(A, B)$ is a homomorphism for any $B$ if, and only if, $\operatorname{dim} A \leq 2$. Also, if $B$ is the self-adjoint part of a finitedimensional nonabelian $C^{*}$-algebra, then every extreme point of $S(A, B)$ is a homomorphism if, and only if, $\operatorname{dim} A \leq 2$.

When $B$ is the real field $\mathbb{R}$, the set $S(A, I R)$ is the state space of $A$ and in this case, every extreme point of $S(A, \mathbb{R})$ is a homomorphism if any only if $S(A, \mathbb{R})$ is a (Choquet) simplex. It is natural to ask whether this is still true for any $B$. The answer is negative. In fact, we will show that $S(A, B)$ is a simplex if and only if either $A=\mathbb{R}$ or $A$ is associative with $B=\mathbb{R}$.

\section{JB-algebras and extreme maps}

We will use [8] as our main reference for $J B$-algebras. In the sequel, by a $J B$-algebra we mean a real Jordan algebra $A$, with identity 1, which is also a Banach space where the Jordan product and the norm are related as follows

$$
\begin{array}{r}
\|a \circ b\| \leq\||a| \mid \cdot\| b \| \\
\|a\|^{2}=\left\|a^{2}\right\| \leq\left\|a^{2}+b^{2}\right\|
\end{array}
$$

for $a, b \in A$. We note that $A$ is partially ordered by the cone $A_{+}=\left\{a^{2}: a \in A\right\}$ and that $A$ is an order-unit normed Banach space with order-unit 1. Moreover, the second dual $A^{\star \star}$ of $A$ is a $J B W$-algebra and $A$ embeds into $A * \star$ as a subalgebra. The self-adjoint part of a unital $C^{*}$-algebra is a $J B$-algebra with the usual Jordan product and the self-adjoint part of a von Neumann algebra is a JBW-algebra.

Let $A$ and $B$ be $J B$-algebras and let $L(A, B)$ be the real Banach space of bounded linear maps from $A$ to $B$. A linear map $\phi: A \rightarrow B$ is positive if $\phi\left(A_{+}\right) \subset B_{+}$. Let $S(A, B)$ be the set of all positive linear 
maps $\phi: A \rightarrow B$ such that $\phi(1)=1$. Then $S(A, B)$ is a convex subset of $L(A, B)$. An extreme point of $S(A, B)$ will be called an extreme map. We note that $S(A, B)$ always contains extreme points. Indeed $S(A, \mathbb{R})=\left\{f \in A^{*}: f(1)=1=\|f\|\right\}$ is the state space of $A$ and it can be embedded as a convex subset of $S(A, B)$ via the map $f \in S(A, I R) \forall \phi_{f} \in S(A, B)$ where $\phi_{f}(a)=f(a) 1_{B}$ for $a \in A$. Moreover, since the states of $B$ separate points of $B$, by composing with the states of $B$, it is easy to see that if $f$ is a pure state of $A$, then $\phi_{f}$ is an extreme point of $S(A, B)$. If $A$ and $B$ are the self-adjoint parts of $C^{*}$ algebras $\underline{A}$ and $\underline{B}$ respectively, we let $\underline{L}(\underline{A}, \underline{B})$ be the space of bounded (complex) linear maps from $\underline{A}$ to $\underline{B}$ and let $\underline{S}(\underline{A}, \underline{B})=\{\phi \in \underline{L}(\underline{A}, \underline{B}): \phi \geq 0, \phi(1)=1\}$ where each map $\phi$ in $\underline{S}(\underline{A}, \underline{B})$ satisfies $\phi\left(a^{*}\right)=\phi(a)^{*}$ for $a \in A$. It follows that the restriction map $\left.\phi \in \underline{S}(\underline{A}, \underline{B}) \mapsto \phi\right|_{A} \in S(A, B)$ is a real affine isomorphism and in particular, the two sets $\underline{S}(\underline{A}, \underline{B})$ and $S(A, B)$ have the same extremal structure.

The following lemma has been proved in [12].

LEMMA 1. Let $A$ be a JB-algebra. Then an element $p$ in $A$ is an extreme point of the positive unit ball $\{a \in A: 0 \leq a \leq 1\}$ if and only if $p$ is a projection, that is, $p^{2}=p$.

A linear map $\phi: A \rightarrow B$ is called a (Jordan) homomorphism if $\phi\left(a^{2}\right)=\phi(a)^{2}$ for all $a$ in $A$. Plainly, every unital Jordan homomorphism is a positive linear map. In fact, it is even an extreme map.

THEOREM 2. If $\phi: A \rightarrow B$ is a unital Jordan homomorphism, then $\phi$ is an extreme point of $S(A, B)$.

Proof. As the second dual map $\phi^{* *}: A^{* *} \rightarrow B^{* *}$ is weakly continuous, it is a Jordan homomorphism by density of $A$ in $A^{* *}$. Suppose that $\phi=\frac{1}{2}(\rho+\psi)$ with $\rho, \psi \in S(A, B)$, then $\phi^{* *}=\frac{z}{2}\left(\rho^{* *}+\psi^{* *}\right)$. Let $e$ be a projection in $A^{* *}$. Then $\phi^{* *}(e)$ is a projection in $B^{* *}$ and hence an extreme point of the positive unit ball in $B^{* *}$, by Lemma 1 . Now $\phi^{* *}(e)=\frac{3}{2} p^{* *}(e)+\frac{1}{2} \psi^{* *}(e)$ and $0 \leq \rho^{* *}(e), \psi^{* *}(e) \leq 1$ imply that 
$\phi^{* *}(e)=\rho^{* *}(e)=\psi^{* *}(e)$. Let $a$ be any element in $A^{* *}$ and let $\varepsilon>0$. By $[8 ; 4,2.3]$, there exist projections $e_{1}, \ldots, e_{n}$ in $A^{\text {:t* }}$ and real numbers $\lambda_{1}, \ldots, \lambda_{n}$ such that

$$
|| a-\sum_{j=1}^{n} \lambda_{j} e_{j}||<\varepsilon
$$

So we have

$$
\begin{aligned}
\left\|\phi^{* *}(a)-\rho^{* *}(a)\right\| & =\left\|\left(\phi^{* *}-\rho^{* *}\right)\left(a-\sum \lambda_{j} e_{j}\right)+\left(\phi^{* *}-\rho^{* *}\right)\left(\sum \lambda_{j} e_{j}\right)\right\| \\
& =\left\|\left(\phi^{* *}-\rho^{* *}\right)\left(a-\Sigma \lambda_{j} e_{j}\right)\right\| \\
& \leq \varepsilon\left\|\phi^{* *}-\rho^{* *}\right\| .
\end{aligned}
$$

This shows that $\phi^{* *}(a)=\rho^{* *}(a)$ for every $a$ in $A^{* *}$. Hence $\phi=\rho$ and $\phi$ is an extreme point of $S(A, B)$.

In general, not every extreme map is a homomorphism as the following lemma shows.

LEMMA 3. Let $A$ be a JB-algebra. The following conditions are equivalent:

(i) $A$ is associative;

(ii) $A$ is isometric isomorphic to the self-adjoint part of an abelian $C^{*}$-algebra;

(iii) the dual cone $A^{*}$ of $A_{+}$is a lattice;

(iv) the state space $S(A, I R)$ is a (Choquet) simplex;

(v) every extreme point of $S(A, \mathbb{R})$ is a Jordan homomorphism.

Proof. (i) $\Rightarrow$ (ii) see $[8 ; 3.2 .2$.$] .$

(iii) $\Rightarrow$ (iv). $S(A, I R)$ is a base of the lattice cone $A^{*}+$ and hence is a simplex (see $[3 ;$ p.138]).

(iv) $\Rightarrow(v)$. Let $f$ be an extreme point of $S(A, I R)$. Then $\{f\}$ is a split face of $S(A, I R)$ (see [3]) and so the kernel $f^{-1}(0)$ is a Jordan ideal in $A$ (see [7; Theorem 2.3], [5; Corollary 3.4]). So $f$ is a Jordan homomorphism. 
(v) $\Rightarrow$ (i). This follows from the fact that the extreme points of $S(A, I R)$ separate points in $A$ and that for each extreme point $f$ in $S(A, I R)$, we have $f\left(\left(\begin{array}{lll}a & \circ & b\end{array}\right) \circ c\right)=f(a) f(b) f(c)=f(a \circ(b \circ c))$ for $a, b, c \varepsilon A$.

Now we study conditions under which the extreme maps are Jordan homomorphisms. As in [8], we define the centre $Z_{A}$ of a $J B$-algebra $A$ to be the set of all elements in $A$ which operator commute with every other element in $A$ where two elements $a$ and $b$ are said to operator commute if $a \circ(c \circ b)=(a \circ c) \circ b$ for all $c$ in $A$. We note that $Z_{A}$ is an associative $J B$-subalgebra of $A$. The following theorem is a straightforward extension of a result of stormer in [10; Theorem 3.1]. We sketch a proof for the sake of completeness.

THEOREM 4. Let $\phi$ be con extreme point of $S(A, B)$. If $a \in Z_{A}$ and $\phi(a) \in Z_{B}$, then $\phi(a \circ b)=\phi(a) \circ \phi(b)$ for $a$ IZ $b \in A$.

Proof. We may assume ||$a||<\frac{1}{2}$, then $\|\phi(a)\|<\frac{1}{2}$. By spectral theory, $\frac{1}{2} 1-a$ and $\frac{1}{2} 1-\phi(a)$ are positive and invertible in $Z_{A}$ and $Z_{B}$ respectively, with $\frac{1}{2} 1-\phi(a) \geq \lambda 1$ for some $\lambda>0$. Define $\psi: A \rightarrow B$ by

$$
\psi(b)=\phi\left(b \circ\left(\frac{1}{2} 1-a\right)\right) \circ\left(\frac{1}{2} 1-\phi(a)\right)^{-1}
$$

for $b \in A$. Then $\psi \in S(A, B)$ and $\lambda \psi \leq \phi$. As $\phi$ is extreme, we have $\psi=\phi$ which gives

$$
\phi(b)=\psi(b)=\phi\left(b o\left(\frac{1}{2} 1-a\right)\right) o\left(\frac{1}{2} 1-\phi(a)\right)^{-1}
$$

and hence $\phi(a \circ b)=\phi(a) \circ \phi(b)$.

Since $B$ is associative if and only if $B=Z_{B}$, the following result follows immediately from Theorem 2 and Theorem 4.

COROLLARY 5. If $B$ is associative and if $\phi$ is an extreme point of $S(A, B)$, then the restriction $\phi \mid Z_{A}$ is an extreme point of $S\left(Z_{A}, B\right)$.

We note that the above result need not be true if $B$ is not associative. We refer to $[10 ; 4.14]$ for an example. Theorem 2 and Theorem 4 also imply the following corollary (see $[1,6,9,10]$ ). 
COROLLARY 6. Let $A$ and $B$ be associative JB-algebras. Then the extreme points of $S(A, B)$ are exactly the unital Jordan homomorphisms from $A$ to $B$.

Let $\ell_{n}^{\infty}$ be the $n$-dimensional abelian $C^{\star}$-algebra of (complex) finite sequences with the minimal projections $e_{1}=(1,0, \ldots, 0)$, $e_{2}=(0,1,0, \ldots, 0), \ldots, e_{n}=(0, \ldots, 0,1)$. We will denote by $\ell_{n}$ the self-adjoint part of $e_{n}^{\infty}$. Let $M_{n}$ be the $C^{*}$-algebra of $n \times n$ complex matrices and let $H_{n}$ be its self-adjoint part consisting of (complex) hermitian matrices. Let $B(H)$ be the full operator algebra on a (complex) Hilbert space $H$. For any projections $p_{1}, \ldots, p_{n}$ in $B(H)$, we say that they are weakly independent if their ranges $p_{1}(H), \cdots, p_{n}(H)$ are weakly independent subspaces of $H$ as defined in $[2 ; \mathrm{p} .165]$, this is equivalent to saying that for any $t_{1}, \ldots, t_{n} \in B(H)$, $\sum_{j=1}^{n} p_{j}{ }_{j} p_{j}=0$ implies $p_{j} t_{j} p_{j}=0$ for $j=1, \ldots, n$ (see $[11 ; \mathrm{p} .102]$ ). We note that if $p$ is a minimal projection in $B(H)$, then for any $t \in B(H), p t p=\lambda p$ for some complex number $\lambda$. Therefore, if $p_{1}, \ldots, p_{n}$ are minimal projections, then they are weakly independent if and only if they are linearly independent. It has been shown in $[2,4,11]$ that a map $\phi$ in $\underline{S}\left(l_{n}^{\infty}, M_{m}^{\prime}\right.$ is an extreme point if and only if the range projections $\operatorname{ran} \phi\left(e_{1}\right), \ldots, \operatorname{ran} \phi\left(e_{n}\right)$ are weakly independent in $M_{m}$.

PROPOSITION 7. Let $B$ be a JB-algebra. Then the extreme points of $S\left(\ell_{2}, B\right)$ are precisely the unital Jordan homomorphisms from $\ell_{2}$ to $B$.

Proof. Let $\phi$ be an extreme point of $S\left(\ell_{2}, B\right)$. To show that $\phi$ is a homomorphism, it suffices to show that $\phi\left(e_{1}\right)$ and $\phi\left(e_{2}\right)$ are projections in $B$ since $1=\phi(1)=\phi\left(e_{1}\right)+\phi\left(e_{2}\right)$. Equivalently, we show that $\phi\left(e_{1}\right)$ and $\phi\left(e_{2}\right)$ are extreme points of the positive unit ball of $B$. Suppose $\phi\left(e_{1}\right)=\frac{b}{2} b+\frac{1}{2} c$ with $0 \leq b, c \leq 1$ in $B$. Define two linear maps $\psi, \rho: \ell_{2} \rightarrow B$ by $\psi\left(e_{1}\right)=b, \psi\left(e_{2}\right)=1-b ; \rho\left(e_{1}\right)=c$ and 
$\rho\left(e_{2}\right)=1-c$. Then clearly $\psi, \rho \in S\left(l_{2}, B\right)$ and $\phi=\frac{1}{2}(\psi+\rho) \cdot$ By extremality of $\phi$, we have $\phi=\psi=\rho$ which gives $b=\psi\left(e_{1}\right)=\rho\left(e_{1}\right)=c$. This proves that $\phi\left(e_{1}\right)$ is an extreme point in the positive unit ball of $B$, so $\phi\left(e_{1}\right)$ is a projection. Likewise $\phi\left(e_{2}\right)$ is also a projection.

The above result is false for $\ell_{n}$ with $n \geq 3$.

Example 1. Define a unital positive (complex) linear map $\phi: \ell_{3}^{\infty} \rightarrow M_{2}$ by

$$
\begin{aligned}
& \phi\left(e_{1}\right)=\left[\begin{array}{rr}
\frac{4}{9} & -\frac{2}{9} \\
-\frac{2}{9} & \frac{1}{9}
\end{array}\right], \quad \phi\left(e_{2}\right)=\left[\begin{array}{rr}
\frac{1}{9} & -\frac{2}{9} \\
-\frac{2}{9} & \frac{4}{9}
\end{array}\right], \\
& \phi\left(e_{3}\right)=\left[\begin{array}{rr}
\frac{4}{9} & \frac{4}{9} \\
\frac{4}{9} & \frac{4}{9}
\end{array}\right] .
\end{aligned}
$$

As $\phi\left(e_{1}\right), \phi\left(e_{2}\right), \phi\left(e_{3}\right)$ are linearly independent and also each $\phi\left(e_{j}\right)$ is a scalar multiple of a minimal projection in $M_{2}$, it follows that the range projections $\operatorname{ran} \phi\left(e_{1}\right), \operatorname{ran} \phi\left(e_{2}\right), \operatorname{ran} \phi\left(e_{3}\right)$ are linearly independent minimal projections which are therefore weakly independent. So $\phi$ is an extreme point of $\underline{S}_{3}\left(\ell_{3}, M_{2}\right)$ by the previous remark. But clearly $\phi$ is not a Jordan homomorphism.

We now consider $S\left(\ell_{n}, B\right)$ for $n \geq 3$. We note that a map $\phi \in S\left(l_{n}, B\right)$ is a Jordan homomorphism if and only if $\phi\left(e_{j}\right)$ is a projection in $B$ for $j=1, \ldots, n$. If $\phi$ is an extreme point of $S\left(l_{n}, B\right)$, the following result shows when $\phi\left(e_{j}\right)$ is 'almost' a projection. 
PROPOSITION 8. Let $\phi$ be con extreme point of $S\left(\ell_{n}, B\right)$. Then $\phi\left(e_{j}\right)^{2} \in \phi\left(l_{n}\right)$ if and only if $\phi\left(e_{j}\right)$ is a scalar multiple of a projection in $B$.

Proof. It suffices to prove the necessity. Suppose $\phi\left(e_{j}\right)^{2} \in \phi\left(\ell_{n}\right)$, then $\phi\left(e_{j}\right)^{2}=\sum_{k=1}^{n} \lambda_{k} \phi\left(e_{k}\right)$ where $\lambda_{k} \in I R$. Without loss of generality we may assume $j=1$. Define $\psi: \ell_{n} \rightarrow B$ by

$$
\psi(a)=a_{1}\left(\lambda_{1} 1_{B}-\phi\left(e_{1}\right)\right) \circ \phi\left(e_{1}\right)+{ }_{k}^{n} \underline{\underline{\Sigma}}_{2} a_{k} \lambda_{k} \phi\left(e_{k}\right)
$$

where $a=\left(a_{1}, \ldots, a_{n}\right) \in \ell_{n}$. Then $\psi(1)=0$ and we have $-\mu \phi \leq \psi \leq \mu \phi$ where $\mu=\max \left\{|| \lambda_{1}-\phi\left(e_{1}\right)||,\left|\lambda_{2}\right|, \ldots,\left|\lambda_{n}\right|\right\}$. Choose $t>0$ such that $t_{\mu} \leq 1$. Let $\phi_{1}=\phi-t \psi$ and $\phi_{2}=\phi+t \psi$. Then we have $\phi_{1}$, $\phi_{2} \in S\left(\ell_{n}, B\right)$ and also $\phi=\frac{1}{2} \phi_{1}+\frac{1}{2} \phi_{2}$. As $\phi$ is an extreme map, we have $\phi=\phi_{1}$ which gives $\left(\lambda_{1}-\phi\left(e_{1}\right)\right.$ ) $0 \phi\left(e_{1}\right)=0$, that is, $\phi\left(e_{1}\right)^{2}=\lambda_{1} \phi\left(e_{1}\right)$. So $\phi\left(e_{1}\right)$ is a scalar multiple of a projection.

From the above result we see that if $\phi$ is an extreme map in $S\left(\ell_{n}, B\right)$ and if each $\phi\left(e_{j}\right)^{2}$ is in $\phi\left(\ell_{n}\right)$ with $\left\|\phi\left(e_{j}\right)\right\|=1$ (or 0$)$, then $\phi$ is a homomorphism. One might conjecture that if an extreme map $\phi: \ell_{n} \rightarrow B$ is such that $\phi\left(\ell_{n}\right)$ is a Jordan algebra, then $\phi$ is a homomorphism. This is false as the following example shows.

Example 2. Define a positive linear map $\phi: \ell_{4} \rightarrow H_{2}$ by

$$
\begin{aligned}
& \phi\left(e_{1}\right)=\frac{1}{5+4 \sqrt{2}}\left[\begin{array}{cc}
4 & -2 \\
-2 & 1
\end{array}\right], \quad \phi\left(e_{2}\right)=\frac{1}{5+4 \sqrt{2}}\left[\begin{array}{cc}
1 & -2 \\
-2 & 4
\end{array}\right], \\
& \phi\left(e_{3}\right)=\frac{2}{5+4 \sqrt{2}}\left[\begin{array}{cc}
\sqrt{2} & 1+i \\
1-i & \sqrt{2}
\end{array}\right], \quad \phi\left(e_{4}\right)=\frac{2}{5+4 \sqrt{2}}\left[\begin{array}{cc}
\sqrt{2} & 1-i \\
1-i & \sqrt{ } 2
\end{array}\right] .
\end{aligned}
$$


Then each $\phi\left(e_{j}\right)$ is a scalar multiple of a minimal projection and as in Example $1, \phi$ is an extreme point of $S\left(\ell_{4}, H_{2}\right)$. Moreover $\phi\left(\ell_{4}\right)=H_{2}$ is a Jordan algebra but $\phi$ is not a Jordan homomorphism.

Actually, if $A$ is a 'nontrivial' $J B$-algebra, then there is always an extreme map $\phi: A \rightarrow H_{2}$ which is not a homomorphism. We have the following result.

THEOREM 9. Let $A$ be a JB-algebra. The following conditions are equivalent:

(i) For any JB-algebra $B$, every extreme point of $S(A, B)$ is a Jordan homomorphism;

(ii) Every extreme point of $S\left(A, H_{2}\right)$ is a Jordan homomorphism;

(iii) $\operatorname{dim} A \leq 2$, that is, $A=\mathbb{R}$ or $\ell_{2}$.

Proof. (ii) $\Rightarrow$ (iii). Let $f$ be a pure state of $A$. Then, as remarked before, the map $\phi_{f}: a \rightarrow f(a)\left[\begin{array}{ll}1 & 0 \\ 0 & 1\end{array}\right]$ is an extreme map in $S\left(A, H_{2}\right)$ and is therefore a Jordan homomorphism. It follows that $f$ is a homomorphism on $A$. Thus, by Lemma $3, A$ is associative and we may assume that $A$ is the self-adjoint part of the $C^{\star}$-algebra $C(X)$ of continuous functions on a compact Hausdorff space $X$. If $\operatorname{dim} A \geq 3$, then $X$ contains three distinct points $x, y, z$ say. Define a (complex) linear map $\phi: C(X) \rightarrow M_{2}$ by

$$
\phi(a)=a(x)\left[\begin{array}{rr}
\frac{4}{9} & -\frac{2}{9} \\
-\frac{2}{9} & \frac{1}{9}
\end{array}\right]+a(y)\left[\begin{array}{rr}
\frac{1}{9} & -\frac{2}{9} \\
-\frac{2}{9} & \frac{4}{9}
\end{array}\right]+a(z)\left[\begin{array}{ll}
\frac{4}{9} & \frac{4}{9} \\
\frac{4}{9} & \frac{4}{9}
\end{array}\right]
$$

for $a \in C(X)$. Then $\phi$ is an extreme point of $\underline{S}\left(C(X), M_{2}\right)$ by weak independence as in Exaraple 1 and by Arveson's theorem in [2; 1. 4. 10]. Now the restriction of $\phi$ to the self-adjoint part $A$ of $C(X)$ is an extreme point of $S\left(A, H_{2}\right)$ and it is not a homomorphism. So $\operatorname{dim} A \leq 2$. 
(iii) $\Rightarrow$ (i). If $\operatorname{dim} A \leq 2$, then $A$ is associative and so $A=\mathbb{R}$ or $\ell_{2}$. If $A=I R$, then $S(A, B)$ is a singleton $\{\Phi\}$ and $\Phi$ is a homomorphism. If $A=\ell_{2}$, then Proposition 7 concludes the proof.

Remark. We see in the above proof that if every extreme point of $S(A, B)$ is a homomorphism, then $A$ is associative. Therefore, if $B$ is associative, then every extreme point of $S(A, B)$ is a homomorphism if and only if $A$ is associative.

To prove our next theorem, we will use the following lema of which the proof is routine and is omitted.

LEMMA 10. Let $A, B$ and $C$ be JB-algebras. Suppose $\phi$ is an extreme map in $S(A, B)$ and $\psi$ an extreme map in $S(A, C)$. Let $\Phi: A \rightarrow B \oplus C$ be defined by $\Phi(a)=\phi(a) \oplus \psi(a)$ for $a \varepsilon A$. Then $\Phi$ is an extreme map in $S(A, B \oplus C)$.

We recall that a type $I$ factor is isomorphic to the full operator algebra $B(H)$ on some Hilbert space $H$. Let $B(H) s \alpha$ be the self-adjoint part of $B(H)$. We will consider $J B$-algebras with a direct summand of $B(H)_{s a}$. We note that an atomic von Neumann algebra is a direct sum of type $I$ factors and a finite-dimensional $C^{*}$-algebra is a finite direct sum of matrix algebras. Moreover, if a von Neumann algebra has a pure normal state, then it contains a direct summand of a type $I$ factor (see $[8 ; 7,5,13]$ ).

THEOREM 11. Let $A$ and $B$ be JB-algebras. Suppose $B$ contains a direct summand of $B(B)_{s a}$ with $\operatorname{dim} H \geq 2$. Then every extreme point of $S(A, B)$ is a Jordan homomorphism if and only if $\operatorname{dim} A \leq 2$.

Proof. The sufficiency follows from Theorem 9. We prove the necessity. So suppose every extreme point in $S(A, B)$ is a homomorphism. By the Remark following Theorem 9, $A$ is associative and we may assume it is the self-adjoint part of some $C(X)$. We need to show $\operatorname{dim} A \leq 2$. If $\operatorname{dim} A \geq 3$, then $X$ contains three distinct points $x, y$ and $z$ say. We deduce a contradiction. By assumption, $B$ contains a direct summand of $B(H)_{s a}$ with $\operatorname{dim} H \geq 2$. So we have $B=B(H)_{s a} \oplus C$ for some $J B$-algebra 
$C$. We first show that there is an extreme point $\phi$ in $S\left(A, B(H){ }_{s a^{\prime}}\right.$ which is not a homomorphism. If $\operatorname{dim} H=2$, such an extreme map exists by Theorem 9. Suppose $\operatorname{dim} H \geq 3$. Define the following positive operators in $B(H)$ :

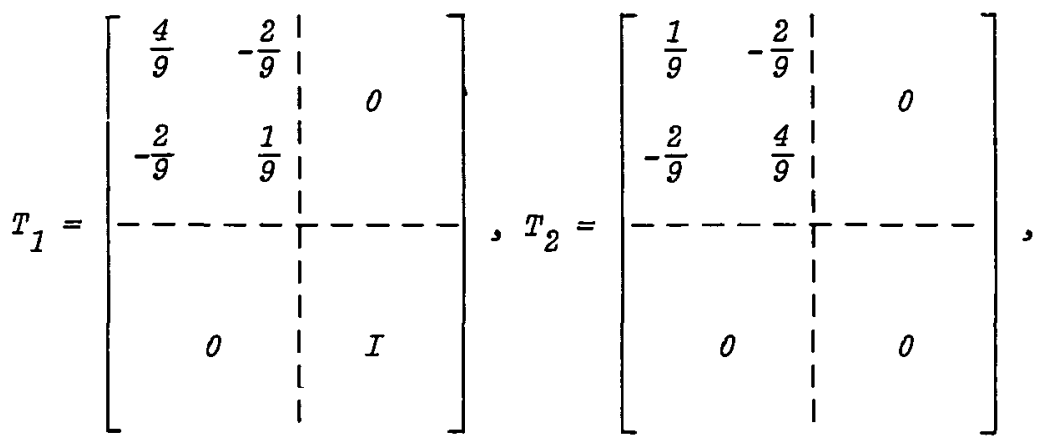

$$
T_{3}=\left[\begin{array}{crc}
\frac{4}{9} & \frac{4}{9} ! & \\
\frac{4}{9} & \frac{4}{9} ! \\
-0 & \vdots & 0 \\
0 & \vdots & 0
\end{array}\right]
$$

where $I$ is the identity operator on a subspace of $H$. Then $T_{1}+T_{2}+T_{3}$ is the identity in $B(H)$ and using Example 1, it can be verified that the range projections $\operatorname{ran} T_{1}, \operatorname{ran} T_{2}, \operatorname{ran} T_{3}$ are weakly independent in $B(H)$. Therefore the linear map $\Phi: C(X) \rightarrow B(H)$ defined by

$$
\Phi(a)=a(x) T_{1}+a(y) T_{2}+a(z) T_{3} \quad(a \in C(X))
$$

is an extreme point of $S(C(X), B(H))$ by Arveson's theorem and its proof in $[2 ; 1.4 .10]$. Evidently $\Phi$ is not a Jordan homomorphism. Hence the restriction of $\Phi$ to the self-adjoint part $A$ of $C(X)$ gives an extreme map $\phi$ in $S\left(A, B(H){ }_{s \alpha^{\prime}}\right.$ which is not a homomorphism. Now let $\rho$ be any 
extreme point of $S(A, C)$. Then by Lemma 10, the map $\psi()=.\phi(.) \oplus \rho($. is an extreme point of $S(A, B(H) s a \oplus C)=S(A, B)$ and also $\psi$ is not a homomorphism. This is a contradiction. So $\operatorname{dim} A \leq 2$. The proof is complete.

Remark. We do not know if the above theorem is true for every non-associative $J B$-algebra $B$.

\section{Simplexes}

We recall that a (non-empty) convex set $S$ in a vector space $E$ is a simplex if for $x \in E$ and $\alpha>0$, the intersection $S n(x+\alpha S)$ is either empty or of the form $y+\beta S$ for some $y \in E$ and $\beta \geq 0$. It is well-known that if $S$ is a base of a cone $K$, then $S$ is a linearly compact simplex if and only if $K$ is a lattice (see [3; p.138]).

Trivially $S(\mathbb{R}, B)$ is a simplex for any $J B$-algebra $B$ since it reduces to a singleton. On the other hand, Lema 3 shows that $S(A, I R)$ is a simplex if and only if $A$ is an associative $J B$-algebra.

THEOREM 12. Let $A$ and $B$ be JB-algebras. The following conditions are equivalent:

(i) $S(A, B)$ is a simplex;

(ii) Either $A=I R$ or $A$ is associative with $B=\mathbb{R}$.

Proof. We only need to prove (i) $\Rightarrow$ (ii). We first show that $A$ is associative. Let $K={ }_{\lambda \geq 0} \lambda S(A, B)$ be the cone generated by $S(A, B)$. Then $K$ is a lattice. We show that the dual cone $A^{*}+$ of $A_{+}$is a lattice. Let $f, g \in A^{*}{ }^{*}$. Define $\phi_{f}, \phi_{g}: A \rightarrow B$ by $\phi_{f}(a)=f(a) 1_{B}$ and $\phi_{g}(a)=g(a) 1_{B}$ for $a \in A$. Then $\phi_{f} \phi_{g} \in K$. So the supremum $\phi=\phi_{f} \vee \phi_{g}$ exists in $K$. Let $h$ be a state of $B$. We show that $h o \phi$ is the lattice supremum of $f$ and $g$. Indeed, for $a \in A_{+}$, we have $(h \circ \phi)(a)=h\left(\left(\phi_{f} \vee \phi_{g}\right)(a)\right) \geq h\left(\phi_{f}(a)\right), h\left(\phi_{g}(a)\right)$ where $h\left(\phi_{f}(a)\right)=f(a)$ and $h\left(\phi_{g}(a)\right)=g(a)$. So $h \circ \phi \geq f, g$. Let $k \in A^{*}$, be such that 
$k \geq f, g$. Let $\phi_{k}: A \rightarrow B$ be the map $\phi_{k}(\cdot)=k(\cdot) I_{B}$. Then we have $\phi_{k} \geq \phi_{f^{\prime}} \phi_{g}$ which implies $\phi_{k} \geq \phi_{f} \vee \phi_{g}$. This in turn implies $k \geq h \circ \phi$. So the supremum $f \vee g$ exists in $A_{+}^{*}$. Hence $A_{+}^{*}$ is a lattice and $A$ is associative by Lemma 3 .

We may now assume that $A$ is the algebra of real continuous functions on some compact Hausdorff space $X$. Suppose $B \neq \mathbb{R}$. Then there exists $b \in B$ such that $0 \leq b \leq 1$ and $b$ is not a scalar multiple of the identity 1. We show that $A=\mathbb{R}$. Suppose, for contradiction, that $A \neq \mathbb{R}$, then there are two distinct points $x$ and $y$ in $X$. The unit masses $\varepsilon_{x}$ and $\varepsilon_{y}$ are pure states of $A$ and can be identified as extreme points of $S(A, B)$ as before. Define $\phi, \psi \in S(A, B)$ by

$$
\begin{aligned}
& \phi(\cdot)=\varepsilon_{x}(\cdot) b+\varepsilon_{y}(\cdot)(1-b) \\
& \psi(\cdot)=\varepsilon_{y}(\cdot) b+\varepsilon_{x}(\cdot)(1-b) .
\end{aligned}
$$

The we have $\frac{1}{2} \phi+\frac{1}{2} \psi=\frac{1}{2} \varepsilon_{x}+\frac{1}{2} \varepsilon_{y}$.

Since $S(A, B)$ is a linearly compact simplex, $\left\{\varepsilon_{x}\right\}$ and $\left\{\varepsilon_{y}\right\}$ are split faces of $S(A, B)$ (see $[3 ; 8.1]$ ) and so the convex hull co $\left\{\varepsilon_{x^{\prime}} \varepsilon y\right\}$ is a (split) face of $S(A, B)$. Now $\frac{1}{2} \phi+\frac{1}{2} \psi=\frac{1}{2} \varepsilon_{x}+\frac{3}{2} \varepsilon_{y} \in$ co $\left\{\varepsilon_{x}, \varepsilon_{y}\right\}$ but $\phi \notin$ co $\left\{\varepsilon_{x}, \varepsilon_{y}\right\}$ since $b \in \phi(A) \neq \mathbb{R} 1_{B}$. This contradicts the fact that co $\left\{\varepsilon_{x}, \varepsilon_{y}\right\}$ is a face. Hence $A=\mathbb{R}$. The proof is complete.

Remark. The above arguments clearly extend to order-unit normed Banach. spaces.

Thus, for example, $S\left(\ell_{2}, \ell_{2}\right)$ is not a simplex while every extreme point of $S\left(\ell_{2}, \ell_{2}\right)$ is a Jordan homomorphism. 


\section{References}

[1] R.F. Arens and J.L. Kelly, "Characterizations of the space of continuous functions over a compact Hausdorff space", Trons. Amer. Math. Soc. 62 (1947) 499-508.

[2] W.B. Arveson, "Subalgebras of C*-algebras", Acta Math. 123 (1969) 141-224.

[3] L. Asimow and A.J. Ellis, Convexity theory and its applications in functional conalysis, (Academic Press, 1980).

[4] M.D. Choi, Positive linear maps on $C^{*}$-algebras, (Thesis, University of Toronto, 1972).

[5] C.M. Edwards, "Ideal theory in JB-algebras", J. London Math. Soc. 16 (1977) 507-513.

[6] A.J. Ellis, "Extreme positive operators", Quart. J. Math. 15 (1964) 342-344.

[7] H. Hanche-Olsen, "Split faces and ideal structure of operator algebras", Math. Scand. 48 (1981) 137-144.

[8] H. Hanche-Olsen and E. Stфrmer, Jordan operator algebras, (Pitman, 1984).

[9] A. Ionescu Tulcea and C. Ionescu Tulcea, "On the lifting property", J. Math. AnaZ. App Z. 3 (1961) 537-546.

[10] E. Stфrmer, "Positive linear maps of operator algebras", Acta Math. 110 (1963) 233-278.

[11] E. Stфrmer, Positive linear mops of $C^{*}$-algebras, Lecture Notes in Physics Vol. 29 (Springer-Verlag, 1974).

[12] J.D.M. Wright and M.A. Youngson, "On isometrics of Jordan algebras", J. London Math. Soc. 17 (1978) 339-344.

Department of Mathematical Sciences

University of London Goldsmiths' College

London SEl4

United kingdom. 